\title{
Quantum Optical Lattices for Emergent Many-Body Phases of Ultracold Atoms
}

\author{
Santiago F. Caballero-Benitez ${ }^{*}$ and Igor B. Mekhov \\ Clarendon Laboratory, Department of Physics, University of Oxford, Parks Road, Oxford OXI 3PU, United Kingdom
}

(Received 18 May 2015; published 11 December 2015)

\begin{abstract}
Confining ultracold gases in cavities creates a paradigm of quantum trapping potentials. We show that this allows us to bridge models with global collective and short-range interactions as novel quantum phases possess properties of both. Some phases appear solely due to quantum light-matter correlations. Because of a global, but spatially structured, interaction, the competition between quantum matter and light waves leads to multimode structures even in single-mode cavities, including delocalized dimers of matter-field coherences (bonds), beyond density orders as supersolids and density waves.
\end{abstract}

DOI: 10.1103/PhysRevLett.115.243604

PACS numbers: 42.50.-p, 03.75.-b, 03.75.Lm, 42.50.Ct

Ultracold atoms trapped in optical lattices (OLs) enable us to study quantum many-body phases with undeniable precision and target problems from several disciplines [1]. Such optical potentials can be complicated, but are prescribed; i.e., they are created by external lasers and are not sensitive to atomic phases. This limits the range of obtainable states. Self-consistent light-matter states can be obtained, when scattered light modifies the trapping potential itself. This was achieved by trapping a BoseEinstein condensate (BEC) inside an optical cavity [2-4], which dramatically enhances the light-matter coupling, thus, making the influence of reemission light comparable to that of external lasers. Such "dynamical potentials" [5] enabled the structural Dicke phase transition and a state with supersolid properties [2]. A key effect observed so far is the dynamical dependence of light intensity (potential depth) on the atomic density. Although the light becomes dynamical, its quantum properties are still not totally exploited, as works on atomic motion in quantum light were limited to a few atoms [6-9]. Effects in dynamical potentials are analogous to semiclassical optics, where atomic excitations are quantum, while light is still classical. As the light and BEC are quantum objects, the quantum fluctuations of both were studied [10,11]; however, the fundamental reason of the structured phase transition can be traced back to the dynamical self-organization predicted [12] and observed [13] with thermal atoms and classical light. For single-mode cavities, dynamical light-matter coupling was shown to lead to several effects [14-18] yet to be observed with bosons and with noninteracting fermions [19-21]. Recently, an optical lattice inside a cavity has been realized [22,23]. Multimode cavities extend the range of quantum phases further [8,24-27].

Published by the American Physical Society under the terms of the Creative Commons Attribution 3.0 License. Further distribution of this work must maintain attribution to the author(s) and the published article's title, journal citation, and DOI.
We show that, even in a single-mode cavity, the quantum potential $[6,28,29]$ leads to significant many-body effects beyond semiclassical ones. Multimode spatial patterns of matter fields arise due to symmetry breaking resulting from the competition between imposed global light structure and standard local processes (tunneling and on-site interactions). We demonstrate that the efficient competition is achieved due to the ability to structure the global interaction at a microscopic scale consistent with the lattice period. Such a competition, in turn, leads to novel many-body states, which are not limited to density-induced orders as in previous studies, but also represent long-range patterns of matter-field coherences (bonds [30]), leading, e.g., to far delocalized dimers, trimers, etc. Importantly, we prove that our approach bridges models with global collective and short-range interactions, as new quantum phases possess properties of both, going beyond Dicke, Lipkin-Meshkov-Glick [31], and other simple spin-1/2 models. Recently, nontrivial spatial patterns were obtained with classical atoms and light [32]. Our Letter will assist in extending such efforts in the interdisciplinary field of optomechanics towards quantum multimode systems [33]. The mechanisms we suggest, provide a general framework and a new set of tools, inaccessible in setups using classical OLs. This will strongly expand applications in quantum simulations. It will allow exploring fundamental issues concerning emergence of multimode generalizations of strongly correlated phases, such as gapped superfluids (SFs) [34] and density waves [35] as well as their interplay, giving rise to quantum solids [36]. The light-induced structure is similar to multicomponent nonlinear sigma models ubiquitous in analog models of high-energy [37,38], condensed matter [39,40], and relativistic [41] physics. Dimer phases can be used as building blocks for quantum spin-liquid simulations [42].

We consider atoms trapped in an OL inside a singlemode cavity with the mode frequency $\omega_{c}$ and decay rate $\kappa$ in off-resonant scattering (see Fig. 1). The pump light with the amplitude $\Omega_{p}$ (in units of the Rabi frequency) and frequency $\omega_{p}\left(\Delta_{c}=\omega_{p}-\omega_{c}\right)$ illuminates atoms in a plane 


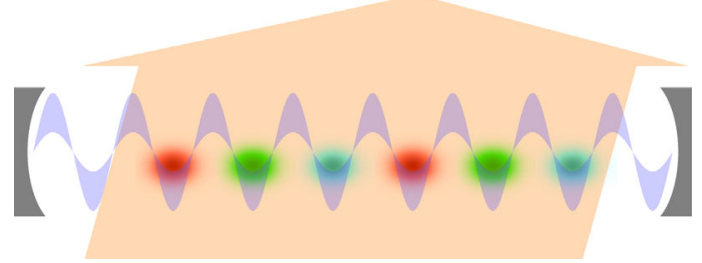

FIG. 1 (color online). Cold atoms trapped in an optical lattice subject to a quantum potential created by the light inside a singlemode cavity. The unsharp potential contour schematically depicts quantum fluctuations of light, which induce the light-matter correlations. The cavity can be a standing or traveling wave. Different colors represent atoms corresponding to different lightinduced spatially structured atomic modes.

transverse to the cavity axis, but not necessarily at $90^{\circ}$. The atoms couple with the cavity mode via the effective coupling strength $g_{2}=g \Omega_{p} /\left(2 \Delta_{a}\right)$, where $g$ is the lightmatter coupling coefficient and $\Delta_{a}$ is the detuning between the light and atomic resonance [29,43-46]. This can be described by the Hamiltonian $\mathcal{H}=\mathcal{H}^{b}+\mathcal{H}^{a}+\mathcal{H}^{a b}$, where $\mathcal{H}^{b}$ is the regular Bose-Hubbard (BH) Hamiltonian [47-49]. The light is described by $\mathcal{H}^{a}=\hbar \omega_{c} \hat{a}^{\dagger} \hat{a}$ and the light-atom interaction is [29]

$$
\mathcal{H}^{a b}=g_{2}^{*} \hat{a} \hat{F}^{\dagger}+g_{2} \hat{a}^{\dagger} \hat{F},
$$

with $\hat{F}=\hat{D}+\hat{B} \cdot \hat{D}=\sum_{j} J_{j, j} \hat{n}_{j}$ is the diagonal coupling of light to on-site densities, $\hat{B}=\sum_{\langle i, j\rangle} J_{i, j}\left(\hat{b}_{i}^{\dagger} \hat{b}_{j}+\right.$ H.c. $)$ is the off-diagonal coupling to the intersite densities reflecting matter-field interference, or bonds [43]. The sums go over illuminated sites $N_{s},\langle i, j\rangle$ denotes nearest neighbor pairs. The operators $\hat{a}^{\dagger}(\hat{a})$ create (annihilate) photons in the cavity, while $b_{i}^{\dagger}\left(\hat{b}_{i}\right)$ corresponds to bosonic atoms at site $i$. $\mathcal{H}^{a b}$ is a consequence of the quantum potential seen by atoms on top of the $\mathrm{BH}$ model given by a classical OL with the hopping amplitude $t_{0}$ and on-site interaction $U$.

The spatial structure of light gives a natural basis to define the atomic modes, as the coupling coefficients $J_{i, j}$ can periodically repeat in space and are calculated from the Wannier functions [51], see [49]. The symmetries broken in the system are inherited from such a periodicity: all atoms equally coupled to light belong to the same mode, while the ones coupled differently belong to different modes. We define operators corresponding to modes $\varphi: \hat{F}=\sum_{\varphi} \hat{D}_{\varphi}+\sum_{\varphi^{\prime}} \hat{B}_{\varphi^{\prime}}$, where

$$
\hat{D}_{\varphi}=J_{D, \varphi} \hat{N}_{\varphi}, \quad \text { with } \quad \hat{N}_{\varphi}=\sum_{i \in \varphi} \hat{n}_{i},
$$

$\hat{B}_{\varphi^{\prime}}=J_{B, \varphi^{\prime}} \hat{S}_{\varphi^{\prime}}, \quad$ with $\quad \hat{S}_{\varphi^{\prime}}=\sum_{\langle i, j\rangle \in \varphi^{\prime}}\left(\hat{b}_{i}^{\dagger} \hat{b}_{j}+\right.$ H.c. $)$.

Thus, we replaced the representation of atomic operator $\hat{F}$ as a sum of microscopic on-site and intersite contributions by the smaller sum of macroscopically occupied global modes with number density, $\hat{N}_{\varphi}$, and bond, $\hat{S}_{\varphi}$, operators.
The structures of density and bond modes can be nearly independent from each other. To be precise, for the homogeneous scattering in a diffraction maximum, $J_{i, j}=$ $J_{B}$ and $J_{j, j}=J_{D}$, one spatial mode is formed. When light is scattered in the main diffraction minimum (at $90^{\circ}$ to the cavity axis), the pattern of light-induced modes alternates sign as in the staggered field, $J_{i, j}=J_{j, i}=(-1)^{j} J_{B}$ and $J_{j, j}=(-1)^{j} J_{D}$. This gives two spatial density modes (odd and even sites) and, as we will show, four bond modes. The density and bond modes can be decoupled by choosing angles such that $J_{D}=0$ (by shifting the probe with respect to the classical lattice, thus, concentrating light between the sites and assuring the zero overlap between Wannier and mode functions) or $J_{B}=0$ [43]. Beyond this, additional modes get imprinted by pumping light at different angles such that each $R$ th site or bond scatters light with equal phases and amplitudes. This generates multimode structures of $R$ density modes [44,52] and $2 R$ bond modes. The prominent example of self-organization [12,15-17] is a special case of two density modes, while macroscopic effects related to the higher density modes and any bond modes have not been addressed so far.

In general, the light and matter are entangled [6,28, 53-56]. In the steady state of light, it can be adiabatically eliminated and the full light-matter state can be then reconstructed as we show in $[49,57]$. The effective atomic Hamiltonian $[6,15,29]$ is

$$
\mathcal{H}_{\text {eff }}^{b}=\mathcal{H}^{b}+\frac{g_{\text {eff }}}{2}\left(\hat{F}^{\dagger} \hat{F}+\hat{F} \hat{F}^{\dagger}\right),
$$

where $g_{\text {eff }}=\Delta_{c}\left|g_{2}\right|^{2} /\left(\Delta_{c}^{2}+\kappa^{2}\right)$. A key physical process is that the ground state is reached [i.e., the energy (4) is minimized], when the system adapts (self-organizes) in such a way that the light scattering term is maximized for $g_{\text {eff }}<0$, and minimized for $g_{\text {eff }}>0$. New terms beyond the BH Hamiltonian give the effective long-range light-induced interaction between density and bond modes

$$
\begin{aligned}
\hat{F}^{\dagger} \hat{F}+\hat{F} \hat{F}^{\dagger}= & \sum_{\varphi, \varphi^{\prime}}\left[\gamma_{\varphi, \varphi^{\prime}}^{D, D} \hat{N}_{\varphi} \hat{N}_{\varphi^{\prime}}+\gamma_{\varphi, \varphi^{\prime}}^{B, B} \hat{S}_{\varphi} \hat{S}_{\varphi^{\prime}}\right. \\
& \left.+\gamma_{\varphi, \varphi^{\prime}}^{D, B}\left(\hat{N}_{\varphi} \hat{S}_{\varphi^{\prime}}+\hat{S}_{\varphi} \hat{N}_{\varphi^{\prime}}\right)\right],
\end{aligned}
$$

where $\gamma_{\varphi, \varphi^{\prime}}^{\nu, \eta}=\left(J_{\nu, \varphi}^{*} J_{\eta, \varphi^{\prime}}+\right.$ c.c. $)$. Thus, any symmetry broken by the light modes imprints the structure on the interaction of atomic modes.

Fundamentally, Eq. (5) displays the link between global interactions and the interaction resembling typical shortrange ones (usually appearing between the sites, while, here, between the modes). Thus, the resulting quantum phase will have properties of both collective and shortrange systems. In this language, it is the term $\hat{N}_{\text {odd }} \hat{N}_{\text {even }}$ that is responsible for supersolid properties of the self-organized state. (The standard supersolidity appears due to $\hat{n}_{i} \hat{n}_{i+1}$ interaction.) Our general approach enables us to go far beyond typical global models (e.g., Dicke and LipkinMeshkov-Glick [31]) due to spatial structuring of the global 
interaction, thus, assuring its effective competition with the short-range ones, even in a single mode cavity in contrast to [8,24-26]. The bond interaction can be easily identified as a pseudospin interaction via the Schwinger mapping [39]. In addition, some components can be non-Abelian.

We decompose (5) in mean-field contributions and fluctuations

$$
\hat{F}^{\dagger} \hat{F}+\hat{F} \hat{F}^{\dagger}=\left\langle\hat{F}^{\dagger}\right\rangle \hat{F}+\langle\hat{F}\rangle \hat{F}^{\dagger}+\delta \hat{F}^{\dagger} \hat{F} .
$$

The last term $\delta \hat{F}^{\dagger} \hat{F}$ originates from the quantum lightmatter correlations, underlying the quantumness of OL. Other terms originate from the dynamical but classical light, when the semiclassical approximation $\hat{a} \hat{F}^{\dagger}=\langle\hat{a}\rangle \hat{F}^{\dagger}$ holds. Decorrelating operators at different sites, we obtain a mean-field theory that has nonlocal coupling between the matter modes and is local in fluctuations. For $\delta \hat{D}^{\dagger} \hat{D}$, these reduce to on-site number fluctuations. Importantly, this corresponds to the purely light-induced effective on-site interaction of atoms beyond the standard $\mathrm{BH}$ term. For $\delta \hat{B}^{\dagger} \hat{B}$, light-matter correlations include radically new terms beyond the $\mathrm{BH}$ model: fluctuations of the order parameter and density coupling between neighboring sites, which appear due to two and four point quantum atomic correlations [49]. In contrast to previous works, we will show non-negligible effects due to such terms, putting forward the quantumness of OLs.

When the ground state of $\mathcal{H}_{\text {eff }}^{b}$ is achieved by maximizing scattering $\left(g_{\text {eff }}<0\right)$, a strong classical light emerges and small fluctuations can be neglected. In principle, even in the strong-light case, the light quantumness can play a role, because self-organized states can be in a superposition of several patterns, and different light amplitudes are correlated to them [6]. Nevertheless, in a realistic case with dissipation, the system quickly collapses to one of the semiclassical states [58]. We will show that quantum fluctuations play a key role in the opposite case, where scattering is minimized $\left(g_{\text {eff }}>0\right)$. Here, no classical light builds up and light fluctuations design the emergence of novel phases. To underline key phenomena, we will consider cases with either density or bond modes.

Scattering at $90^{\circ}$, one breaks the translational symmetry. Hence, the system can support density waves (DWs) and novel bond orders. The simultaneous occurrence of SF and DW orders is a supersolid (SS) phase [36]. SSs and DWs have been predicted due to classical maximized scattering $[16,17]$. We show that for a weak pump, DWs and SSs with only small density imbalance appear at half-integer filling, together with the usual Mott insulator (MI) and SF $[22,49]$. In contrast, above the threshold $\left|g_{\text {eff }}\right| N_{s}>U / 2$, we find that DWs and SSs with maximal imbalance are favored, while the usual MI and SF are completely suppressed $[22,49]$.

For minimized scattering $\left(g_{\text {eff }}>0\right)$, the classical light cannot build up at all, and quantum fluctuations take the leading role [Fig. 2(a)]. At fixed density per site, the quantum light-matter fluctuations effectively renormalize the on-site interaction from $U$ to $U+2 g_{\text {eff }} J_{D}^{2}$ in each component. Thus, changing the light-matter coupling, one can shift the SF-MI transition point. This occurs because the light-induced atomic fluctuations now enter the effective Hamiltonian, and these fluctuations need to be suppressed to minimize the energy. This favors the MI state for $U$ smaller than that without cavity light, extending MI regions [Fig. 2(a)]. For incommensurate fillings, SF survives but with smaller (suppressed) fluctuations as well: For convenience, we also plot the boundaries where the superfluid ground state is composed of mainly the two lowest occupation Fock states depending on the filling factor (i.e., components with higher occupations are negligible). Note that, with cavity light, the state becomes gapped with respect to adding more than one excitation, thus, minimizing fluctuations. However, there is no phase transition to this peculiar superfluid state [49]. Moreover, in a quantum OL, atoms can potentially enter MI even without atomic interaction. This provides absolute control on DW order formation. In analogous fermionic systems, DWs are relevant for the stability of superconducting phases [61].

Scattering at angles different from $90^{\circ}$ creates more than $R=2$ atomic modes [44,52], and the light-imposed coefficients are $J_{j, j}=J_{D} \chi(j)$, where, for traveling waves, $\chi(j)=\exp (i 2 \pi j / R)$. Now, multiple terms $\hat{N}_{\varphi} \hat{N}_{\varphi^{\prime}}$ in Eq. (5) become important. We present a phase diagram for various scattering angles (inducing $R$ modes) for strong
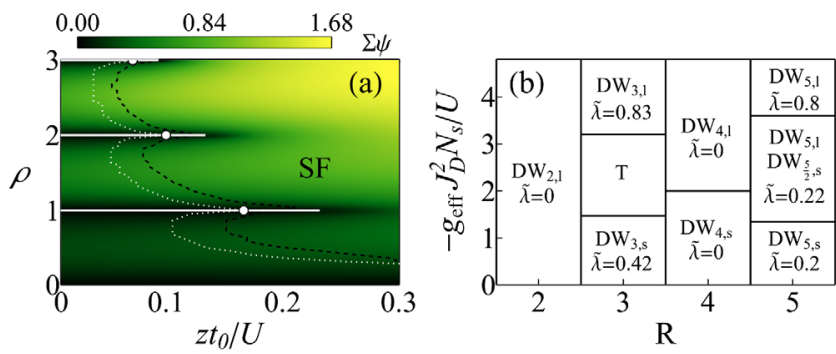

FIG. 2 (color online). Light scattering from on-site densities. (a) Minimized scattering highlighting the quantumness of OL. Total order parameter $\Sigma \psi=\left|\psi_{+}\right|^{2}+\left|\psi_{-}\right|^{2}$, where $\psi_{ \pm}$are the SF order parameters of two modes, white lines correspond to MIs; dashed lines are boundaries of SF with only two non-negligible Fock components. The system is homogeneous, $\rho_{+}=\rho_{-}$and $\psi_{+}=\psi_{-}$. White points are the MI-SF transition points without cavity light, the SF-MI can be significantly shifted by the quantum OL. (b) Strongly interacting phase diagram for multiple number of modes $R$ (created for different pump angles) at half filling. Quantum phases have a period of density-density correlations $q$ (in units of lattice period), $\mathrm{DW}_{q, l}\left(\mathrm{DW}_{q, s}\right), l(s)$ denotes large (small) density imbalance $\mathrm{DW}, \tilde{\lambda}$ is the SF fraction $[49,59,60]$. DW and SF order depend on the effective light-matter interaction strength $g_{\text {eff }}$ and $R$. Horizontal lines denote phase boundaries between quantum phases. Parameters: (a) scattering at $90^{\circ}, g_{\text {eff }}=10 U / N_{s}$, the boundaries are for $g_{\text {eff }}=10 U / N_{s}$ and $0 ; J_{D}=1.0, J_{B}=0, N_{s}=100, z=6$.(b) scattering at various angles defining the mode number $R, J_{D}=1.0$, $J_{B}=0, t_{0}=0, N_{s}=100$. 
on-site interaction with maximal light scattering [Fig. 2(b)]. The $R$-mode induced pattern competes with on-site interaction modifying the density distribution. Therefore, multiple DWs of period $R$ can coexist with SF, forming multicomponent SSs. Surprisingly, at half-filling for $R>2$, odd, SS exists. As $\left|g_{\text {eff }}\right|$ changes from zero, checkerboard insulators form for even $R$ while different kinds of SSs exist for odd $R$. The on-site interaction limits atomic fluctuations producing gapped SF components when SS exists. As $R$ increases, additional DWs with different periods and amplitudes emerge. These form unstable mixed state configurations $(T)$ for $R=3$ and stable multicomponent SSs for $R=5$. These occur in between regular SS phases with DWs of period $R$ and different amplitude. As the light induces atomic fluctuations, these generate competition between small and large amplitude DWs until saturation in the SF component occurs. The system reaches a configuration, similar to the maximal imbalance DW state described above for scattering at $90^{\circ}$.

We find a novel phase transition, when light scattering from the bonds at $90^{\circ}\left[J_{j, j+1}=(-1)^{j} J_{B}, J_{D}=0\right]$ is maximized $\left(g_{\text {eff }}<0\right)$. Even in the absence of on-site interaction, a transition from normal SF to the superfluid dimer (SFD) state appears. SFD is a SF state in which the complex order parameter has alternating (zero and nonzero) phase difference between pairs of sites, and its amplitude is modulated as well. This occurs because of the competition between the kinetic energy $\mathrm{BH}$ terms, which promote a homogeneous SF, with the light-induced interaction that favors SF components with alternating phases across every other site [Fig. 3(a)]. The phase of light interference flips from bond to bond [such that the phase difference at neighboring bonds is $\pi$, Fig. 3(a)]. In the Hamiltonian, this corresponds to alternating signs in front of matter-field coherences between the neighboring sites (i.e., products of complex order parameters, in mean-field treatment). To minimize the energy (and maximize the light scattering) the quantum matter fields self-organize such that the matterfield phase difference between neighboring sites flips as well to compensate for the imposed light-field phase flip. The dimer configurations have high degeneracy as the full many-body ground-state is composed of several equivalent arrangements of the phase pattern in space [49]. The phase diagram is shown in Fig. 3(b). Moreover, in the presence of on-site interaction, the system supports a transition to the supersolid dimer (SSD) state with modulated densities [Fig. 3(c)]. The on-site interaction suppresses atomic fluctuations, while, as light scattering gets optimized, the density is unable to lock in a homogeneous density pattern leading to additional density imbalance. Therefore, the phase modulation and density modulations coexist simultaneously while atoms retain mobility preventing the stabilization of an insulating phase. Note that multimode bond structures can have very nontrivial spatial overlap and dimers (and their multimode generalizations as trimers,

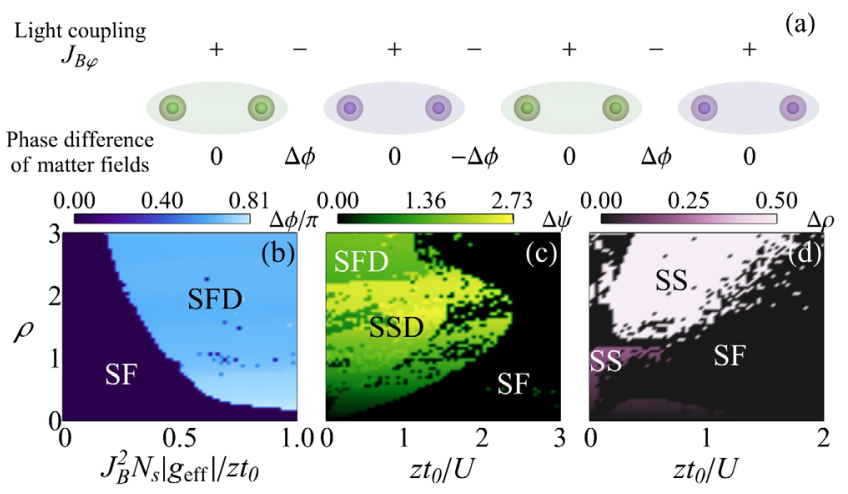

FIG. 3 (color online). Emergent dimer phases and quantumlight-induced supersolids due to scattering from bonds. (a) Dimer structure for maximized scattering: matter-field coherence compensates for imposed light-field patterns. (b) Phase diagram for the phase difference $\Delta \phi$ between dimers when light scattering is maximized without on-site interaction. (c) Phase diagram for the difference in order parameters $\psi_{A / B}$ of the dimers $\Delta \psi=\left.|| \psi_{A}\right|^{2}-$ $\left|\psi_{B}\right|^{2} \mid / 2$ when light scattering is maximized with on-site interaction. (d) Density wave order parameter $\Delta \rho=\left|\rho_{A}-\rho_{B}\right| / 2$ for minimized scattering. The density components $\rho_{A / B}$ correspond to atomic populations of effective light-induced modes. Regions with $\Delta \rho \neq 0$ correspond to supersolid phases; $\Delta \rho=0$ corresponds to superfluid. Parameters: (b) $U=0, \quad$ (c) $g_{\text {eff }}=-25 U / N_{s}$, (d) $g_{\text {eff }}=25 U / N_{s} ; J_{D}=0, J_{B}=0.1, N_{s}=100$, and $z=6$.

tetramers, etc., which can be obtained for a tilted pump angle) extend over many sites demonstrating the interplay of global and short-range properties. Dimer states can be used as fundamental units to engineer Hamiltonians of quantum spin-liquid states [42]. As $\hat{B}^{\dagger} \hat{B}$ processes enter directly in the effective Hamiltonian with couplings depending on the light geometry and pump amplitude, this makes it feasible to achieve analogous physics, relaxing the constraint on very low temperatures and large on-site interactions based on second order expansion effective Hamiltonians [1].

Importantly, we prove that there is a SS to SF transition that is solely driven by quantum correlations for minimized light scattering $\left(g_{\text {eff }}>0\right)$, Fig. 3(d). This occurs because the terms due to light-matter correlations in $\hat{B}^{\dagger} \hat{B}$ are not shadowed by semiclassical effects, as there is no classical light build up. Two-point tunneling correlations introduce new terms in the BH model [49], which couple densities at neighboring sites only: $\sum_{\langle i, j\rangle} \hat{n}_{i} \hat{n}_{j}$, producing a DW instability even without strong light. Density imbalance is energetically favored and the atoms condense in a nearest-neighbor density pattern, while additional terms in $\mathcal{H}_{\text {eff }}^{b}$ favor atomic quantum fluctuations competing with the onsite interaction. Thus, short range processes induced by the quantumness of light induce the transition. Such a direct density coupling corresponds stronger to the typical supersolidity scenario, which is under active search $[36,62]$.

In experiments with homogeneous BECs, both $\kappa$ and the long-range interaction rate are of the same order (either 
MHz [63] or $\mathrm{kHz}$ [64]), while the depletion rate [65] of the ground state can be made smaller by choosing the detuning such that $\left|\kappa / \Delta_{c}\right|<1$. In our lattice case, the effective lightmatter coupling coefficient $g$ rescales as $g J_{D, B}$ and, similar to the homogeneous systems, the evolution can be faster than depletion.

In conclusion, we have shown that quantum optical lattices offer a new tool to engineer nonlocal many-body interactions with light-induced structures. These interactions can break symmetries by design and imprint a pattern that governs the origin of many-body phases. This effectively bridges physics of long-range and short-range interactions. The light and matter are entangled, forming nontrivial light-matter correlated states. We suggested how to generate not only multimode density patterns, but nonlocal patterns of matter-filed coherences as well (in particular, delocalized superfluid and supersolid dimers). Some of the states appear solely due to quantum fluctuations of light and matter, where no classical light can build up. A pathway for realizing our proposal is to combine several recent experimental breakthroughs: a BEC was trapped in a cavity, but without a lattice [2-4], and detection of light scattered from ultracold atoms in an OL was performed, but without a cavity $[66,67]$. Very recently, an optical lattice in a cavity became a reality $[22,23]$. Based on off-resonant scattering and, thus, being nonsensitive to a detailed atomic level structure, our approach can be extended to other arrays of natural or artificial quantum objects: spins, fermions, molecules (including biological ones) [68], ions [69], atoms in multiple cavities [70], and semiconductor [71] or superconducting qubits [72].

The work was supported by the EPSRC (Grant No. EP/I004394/1).

* santiago.caballerobenitez@physics.ox.ac.uk

[1] M. Lewenstein, A. Sampera, and V. Ahufinger, Ultracold Atoms in Optical Lattices: Simulating Quantum Many-Body Systems. (Oxford University Press, New York, 2012).

[2] K. Baumann, C. Guerlin, F. Brennecke, and T. Esslinger, Nature (London) 464, 1301 (2010).

[3] M. Wolke, J. Klinner, H. Kessler, and A. Hemmerich, Science 337, 75 (2012).

[4] D. Schmidt, H. Tomczyk, S. Slama, and C. Zimmermann, Phys. Rev. Lett. 112, 115302 (2014).

[5] H. Ritsch, P. Domokos, F. Brennecke, and T Esslinger, Rev. Mod. Phys. 85, 553 (2013).

[6] C. Maschler, I. B. Mekhov, and H. Ritsch, Eur. Phys. J. D 46, 545 (2008).

[7] A. Vukics, C. Maschler, and H. Ritsch, New J. Phys. 9, 255 (2007).

[8] S. Krämer and H. Ritsch, Phys. Rev. A 90, 033833 (2014).

[9] D. J. Winterauer, W. Niedenzu, and H. Ritsch, Phys. Rev. A 91, 053829 (2015).

[10] G. Kónya, G. Szirmai, and P. Domokos, Phys. Rev. A 90, 013623 (2014).
[11] R. Landig, F. Brennecke, R. Mottl, T. Donner, and T. Esslinger, Nat. Commun. 6, 7046 (2015).

[12] P. Domokos and H. Ritsch, Phys. Rev. Lett. 89, 253003 (2002).

[13] A. T. Black, H. W. Chan, and V. Vuletić, Phys. Rev. Lett. 91, 203001 (2003).

[14] J. Larson, B. Damski, G. Morigi, and M. Lewenstein, Phys. Rev. Lett. 100, 050401 (2008).

[15] S. Fernandez-Vidal, G. De Chiara, J. Larson, and G. Morigi, Phys. Rev. A 81, 043407 (2010).

[16] Y. Li, L. He, and W. Hofstetter, Phys. Rev. A 87, 051604(R) (2013).

[17] M. R. Bakhtiari, A. Hemmerich, H. Ritsch, and M. Thorwart, Phys. Rev. Lett. 114, 123601 (2015).

[18] H. Habibian, A. Winter, S. Paganelli, H. Rieger, and G. Morigi, Phys. Rev. Lett. 110, 075304 (2013).

[19] J. Keeling, M. J. Bhaseen, and B. D. Simons, Phys. Rev. Lett. 112, 143002 (2014).

[20] F. Piazza and P. Strack, Phys. Rev. Lett. 112, 143003 (2014).

[21] Y. Chen, Z. Yu, and H. Zhai, Phys. Rev. Lett. 112, 143004 (2014).

[22] R. Landig, L. Hruby, N. Dogra, M. Landini, R. Mottl, T. Donner, and T. Esslinger, arXiv:1511.00007.

[23] J. Klinder, H. Keßler, M. R. Bakhtiari, M. Thorwart, and A. Hemmerich, arXiv:1511.00850.

[24] S. Gopalakrishnan, B. S. Lev, and P. M. Goldbarth, Nat. Phys. 5, 845 (2009).

[25] P. Strack and S. Sachdev, Phys. Rev. Lett. 107, 277202 (2011).

[26] M. Müller, P. Strack, and S. Sachdev, Phys. Rev. A 86, 023604 (2012).

[27] A. J. Kollár, A. T. Papageorge, K. Baumann, M. A. Armen, and B. L. Lev, New J. Phys. 17, 043012 (2015).

[28] I. B. Mekhov, C. Maschler, and H. Ritsch, Nat. Phys. 3, 319 (2007).

[29] I. B. Mekhov and H. Ritsch, J. Phys. B 45, 102001(2012).

[30] L. Bonnes, D. Charrier, and A. M. Läuchli, Phys. Rev. A 90, 033612 (2014).

[31] S. Morrison and A. S. Parkins, Phys. Rev. Lett. 100, 040403 (2008).

[32] G. Labeyrie, E. Tesio, P. M. Gomes, G.-L. Oppo, W. J. Firth, G. R. M. Robb, A. S. Arnold, R. Kaiser, and T. Ackemann, Nat. Photonics 8, 321 (2014).

[33] M. Aspelmeyer, T. J. Kippenberg, and F. Marquardt, Rev. Mod. Phys. 86, 1391 (2014).

[34] B. Zeng and X.-G. Wen, Phys. Rev. B 91, 125121 (2015).

[35] G. Grüner, Rev. Mod. Phys. 60, 1129 (1988).

[36] F. Cinti, T. Macrí, W. Lechner, G. Pupillo, and T. Pohl, Nat. Commun. 5, 02 (2014).

[37] E. Zohar, J. I. Cirac, and B. Reznik, Phys. Rev. Lett. 110, 125304 (2013).

[38] K. Stannigel, P. Hauke, D. Marcos, M. Hafezi, S. Diehl, M. Dalmonte, and P. Zoller, Phys. Rev. Lett. 112, 120406 (2014).

[39] A. Auerbach, Interacting Electrons and Quantum Magnetism (Springer-Verlag, New York, 1994).

[40] I. Bloch, J. Dalibard, and J. Sylvain, Nat. Phys. 8, 267 (2012).

[41] E. Witten, Nucl. Phys. B223, 422 (1983).

[42] L. Balents, Nature (London) 464, 199 (2010). 
[43] W. Kozlowski, S. F. Caballero-Benitez, and I. B. Mekhov, Phys. Rev. A 92, 013613 (2015).

[44] G. Mazzucchi, W. Kozlowski, S. F. Caballero-Benitez, T. J. Elliott, and I. B. Mekhov, arXiv:1503.08710.

[45] G. Mazzucchi, S. F. Caballero-Benitez, and I. B. Mekhov, arXiv: 1510.04883.

[46] W. Kozlowski, S. F. Caballero-Benitez, and I. B. Mekhov, arXiv: 1510.04857.

[47] M. P. A. Fisher, P. B. Weichman, G. Grinstein, and D. S. Fisher, Phys. Rev. B 40, 546 (1989).

[48] D. Jaksch, C. Bruder, J. I. Cirac, C. W. Gardiner, and P. Zoller, Phys. Rev. Lett. 81, 3108 (1998).

[49] See Supplemental Material at http://link.aps.org/ supplemental/10.1103/PhysRevLett.115.243604 for details on the effective Hamiltonians, quantum many-body phases, the full-light matter quantum state and numerical simulations, which includes Ref. [50].

[50] P. Bak, Phys. Today 39, No. 12, 38 (1986).

[51] R. Walters, G. Cotugno, T. H. Johnson, S. R. Clark, and D. Jaksch, Phys. Rev. A 87, 043613 (2013).

[52] T. J. Elliott, W. Kozlowski, S. F. Caballero-Benitez, and I. B. Mekhov, Phys. Rev. Lett. 114, 113604 (2015).

[53] I. B. Mekhov and H. Ritsch, Phys. Rev. A 80, 013604 (2009).

[54] I. B. Mekhov and H. Ritsch, Laser Phys. 19, 610 (2009).

[55] I. B. Mekhov and H. Ritsch, Laser Phys. 20, 694 (2010).

[56] I. B. Mekhov and H. Ritsch, Laser Phys. 21, 1486 (2011).

[57] S. F. Caballero-Benitez and I. B. Mekhov, arXiv:1508.05640 [New J. Phys. (to be published)].

[58] K. Baumann, R. Mottl, F. Brennecke, and T. Esslinger, Phys. Rev. Lett. 107, 140402 (2011).
[59] G. D. Mahan, Many Particle Physics, 3rd ed. (Plenum, New York, 2000).

[60] C. N. Yang, Rev. Mod. Phys. 34, 694 (1962).

[61] E. H. da Silva Neto et al., Science 343, 393 (2014).

[62] S. Baier, M. J. Mark, D. Petter, K. Aikawa, L. Chomaz, Z. Cai, M. Baranov, P. Zoller, and F. Ferlaino, arXiv:1507.03500.

[63] F. Brennecke, R. Mottl, K. Baumann, R. Landig, T. Donner, and T. Esslinger, Proc. Natl. Acad. Sci. U.S.A. 110, 11763 (2013).

[64] J. Klinder, H. Keßler, M. Wolke, L. Mathey, and A. Hemmerich, Proc. Natl. Acad. Sci. U.S.A. 112, 3290 (2015).

[65] D. Nagy, G. Konya, G. Szirmai, and P. Domokos, Phys. Rev. Lett. 104, 130401 (2010).

[66] C. Weitenberg, P. Schauß, T. Fukuhara, M. Cheneau, M. Endres, I. Bloch, and S. Kuhr, Phys. Rev. Lett. 106, 215301 (2011).

[67] H. Miyake, G. A. Siviloglou, G. Puentes, D. E. Pritchard, W. Ketterle, and D. M. Weld, Phys. Rev. Lett. 107, 175302 (2011).

[68] I. B. Mekhov, Laser Phys. 23, 015501 (2013).

[69] R. Blatt and C. F. Roos, Nat. Phys. 8, 277 (2012).

[70] M. Hartmann, F. Brandao, and M. B. Plenio, Nat. Phys. 2, 849 (2006).

[71] B. Trauzettel, D. V. Bulaev, D. Loss, and G. Burkards, Nat. Phys. 3, 192 (2007).

[72] J. M. Fink, R. Bianchetti, M. Baur, M. Göppl, L. Steffen, S. Filipp, P. J. Leek, A. Blais, and A. Wallraff, Phys. Rev. Lett. 103, 083601 (2009). 